\title{
APPLICATION OF GENERALIZED PAIR APPROXIMATION TO DILUTED MAGNETIC SEMICONDUCTOR QUANTUM STRUCTURES
}

\author{
H. Bednarski ${ }^{a, b}$, J. Cisowski ${ }^{a, c}$ and J.C. Portal ${ }^{b}$ \\ ${ }^{a}$ Department of Solid State Physics, Polish Academy of Sciences \\ Wandy 3, 41-800 Zabrze, Poland \\ ${ }^{b}$ Laboratoire des Champs Magnétiques Intenses, CNRS, Grenoble, France \\ 'Institute of Physics, Silesian Technical University \\ Bolesława Krzywoustego 2, 44-100 Gliwice, Poland
}

\begin{abstract}
Analysing the $d-d$ interaction in diluted magnetic semiconductor quantum structures, we used our generalized pair approximation which permits to take into account the real spatial distribution of the magnetic ions in diluted magnetic semiconductor layers close to the nonmagnetic quantum well. We subsequently applied our approach to calculate the Zeeman splittings in the $\mathrm{CdTe} / \mathrm{Cd}_{1-x} \mathrm{Mn}_{x} \mathrm{Te}$ quantum structures with $x=5$ and $7.5 \%$ and we obtained a good agreement with the experimental results.
\end{abstract}

PACS numbers: $71.70 . \mathrm{Ej}, 75.30 . \mathrm{Et}$

As is known, an external magnetic field acting upon a diluted magnetic semiconductor (DMS) produces a giant Zeeman splitting of both the conduction and valence bands. The magnitude of the observed changes in energy of the spin states of the bands is proportional to the average value of the magnetic ions spin $\left\langle S_{z}\right\rangle$ described usually by the modified Brillouin function. Such an approach is justified, in principle, for bulk materials in which the random and homogeneous spatial distribution of the magnetic ions may be assumed. In DMS quantum structures (QS), especially those containing a nonmagnetic semiconducting well surrounded by DMS barriers, the spatial homogeneous distribution of the magnetic ions is intentionally broken by technological manufacturing which produces an enhancement in paramagnetism associated with the interface layers. Nevertheless, the modified Brillouin function remains very useful in description of DMS QS even with high magnetic ions concentration [1].

The purpose of our paper is to present a theoretical model for a more adequate description of the magneto-optical effects in DMS QS for moderate magnetic ion concentrations $(x \leq 10 \%)$. Our method is based on the generalized pair approximation (GPA) [2] originally applicable to bulk DMS in which the magnetic ions may occupy a few kinds of inequivalent sites of the crystal lattice. 
A DMS perfect quantum well (QW) can be considered as a solid consisting of three regions, i.e. DMS-semiconductor (SC)-DMS with the perfect DMS-SC interfaces; for simplicity we also assume that the magnetic ions concentration is the same in both DMS layers. From the point of view of the GPA, such a structure is a solid with inequivalent planes perpendicular to the growth direction. It means that all ions belonging to a given plane have the same numbers of first $(1 \mathrm{~N})$, second $(2 \mathrm{~N})$, third $(3 \mathrm{~N})$, etc., neighbors, so they are all equivalent but the ion sites belonging to different planes may be inequivalent. For example, all the ions placed in DMS in the plane adjacent to the nonmagnetic SC have $8(1 \mathrm{~N}), 5(2 \mathrm{~N})$, $12(3 \mathrm{~N})$, etc., while all the ions in the consecutive DMS plane have $12,5,20$, etc., neighbors, respectively. Thus, within the GPA, the average spin of Mn ions $\left\langle S_{\alpha}(x)\right\rangle$ for a given plane $\alpha$ of a perfect DMS QW can be written as

$$
\left\langle S_{\alpha}(x)\right\rangle=\frac{N_{\alpha}}{2 g \mu_{\mathrm{B}}} \sum_{\nu}^{\infty} P_{\nu}^{\alpha}(x) \frac{\partial F_{\nu}\left(J\left(R_{\nu}\right)\right)}{\partial B},
$$

where $N_{\alpha}$ is the total number of spins in the $\alpha$ plane, $g$ is the Lande factor of the ions, $\mu_{\mathrm{B}}$ is the Bohr magneton, $F_{\nu}$ is the free energy of a pair of interacting spins, $J\left(R_{\nu}\right)$ is an exchange constant between these spins separated by a distance $R_{\nu}$ and $P_{\nu}^{\alpha}(x)$ is the probability of finding such a pair.

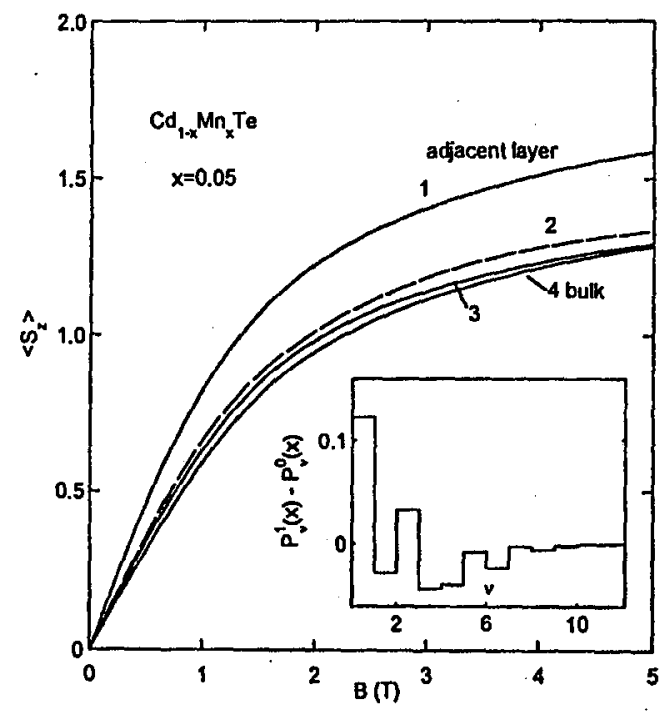

Fig. 1. Magnetic field dependence of the averaged spin of $\mathrm{Mn}^{2+}$ ions in the adjacent (1) and the second (3) to the semiconductor well $\mathrm{Cd}_{0.95} \mathrm{Mn}_{0.05} \mathrm{Te}$ layers, as compared with the bulk (4). Solid lines $(1,3,4)$ are calculated according to Eq. (1) with the first neighbor exchange constant $J_{1}=-7.2 \mathrm{~K}[5]$ and the dashed line (2) represents the result for the bulk with $J_{1}=-5.5 \mathrm{~K}$. The inset shows the difference in probability of finding a pair of interacting $\mathrm{Mn}$ ions separated by a distance $R_{\nu}$ in the adjacent and second layers. 
Using such an approach, we can now visualize the enhancement of paramagnetism which is also called the interface magnetic correction effect [1]. The averaged spin of magnetic ions in several first planes of DMS/SC interface is shown in Fig. 1. As can be seen, the enhancement in paramagnetism occurs only for the first magnetic monolayer adjacent to the nonmagnetic well. One can easy understand the origin of this phenomenon by comparing the appropriate probabilities for finding two interacting magnetic ions separated by the same distance $R_{\nu}$ but belonging to different planes, as shown in the inset of Fig. 1. As is clearly seen, the main contribution to this effect comes from a difference between the numbers of the nearest-neighbor pairs in the adjacent and the second monolayer to the $\mathrm{QW}$ and that the averaged spin of the latter is only slightly higher than in the bulk.

We can now apply our method to calculate the Zeeman splitting in two slightly different $\mathrm{CdTe} / \mathrm{Cd}_{1-x} \mathrm{Mn}_{x} \mathrm{Te}$ multiple $\mathrm{QW}$ structures for which there exist the experimental data on the photoluminescence excitation spectroscopy in the Voigt geometry and at $T \leq 2 \mathrm{~K}[3,4]$. The first DMS QS consists of $8 \mathrm{CdTe}$ wells of $78 \AA$ width separated by $200 \AA \mathrm{Cd}_{0.95} \mathrm{Mn}_{0.05} \mathrm{Te}$ barriers [3], while the second one is built up of 15 wells of $75 \AA$ width and $158 \AA \mathrm{Cd}_{0.925} \mathrm{Mn}_{0.075} \mathrm{Te}$ barriers [4] and both structures are grown on $\operatorname{InSb}(100)$ substrates.

In the first step, the Zeeman splitting for a perfect (rectangular) DMS QW with the $d-d$ exchange interaction of the form $J\left(R_{\nu}\right)=J_{1} R_{\nu}^{-6.8}$ [5], using the method described in $[3,6]$ and with all other parameters which are the same as in [3], has been calculated with the only one fitting parameter $J_{1}=-5.5 \mathrm{~K}$, instead of two, when using the modified Brillouin function. Ilowever, this value of $1 \mathrm{~N} \mathrm{ex-}$ change interaction differs significantly from that known for the bulk $\mathrm{Cd}_{1-x} \mathrm{Mn}_{x} \mathrm{Te}$ $\left(J_{1}=-7.2 \mathrm{~K}\right)[5]$. Thus, in the second step we have taken into account the real structure of the DMS QW characterized by the existence of finite regions with the different $\mathrm{Mn}$ concentration (the interface mixing) [1,7]. As follows from the detailed quantitative description of the Zeeman splittings of excitonic states in $\mathrm{CdTe} / \mathrm{Cd}_{1-x} \mathrm{Mn}_{x} \mathrm{Te}$ systems with higher $(x>0.15)$ concentration of magnetic ions [1], the interface mixing is more important than the interface magnetic correction. Extending this description towards smaller $\mathrm{Mn}$ concentration and applying the GPA, we have calculated the energies of magneto-optical transitions originating from both the heavy hole and light hole subbands to the conduction subbands.

The final results of our calculations are shown in Fig. 2 as solid lines, giving a good description of the experimental data $[3,4]$. The value of the exchange interaction constant used in these calculations is the same as that cited above, i.e. $J_{1}=-7.2 \mathrm{~K}[5]$ and the valence band offset relative to the band gap difference ("chemical offset") has been found from the fitting procedure as being equal to 0.375 . During calculations we have assumed the $20 \%$ interface disorder on the two monolayers (ML) scale which means that $2 \mathrm{ML}$ in the well region adjacent to the interface contain $0.2 x \mathrm{Mn}$, whereas $2 \mathrm{ML}$ in the barrier region adjacent to the interface have $0.8 x \mathrm{Mn}$ and we believe that this simplifying assumption is responsible for small remaining discrepancies between our approach and experiment.

Our paper shows that the generalized pair approximation, which is very useful for description of the $d-d$ interaction in bulk DMS possessing a few inequivalent magnetic ion sites [2], can be also applied to real diluted magnetic scmiconductor 

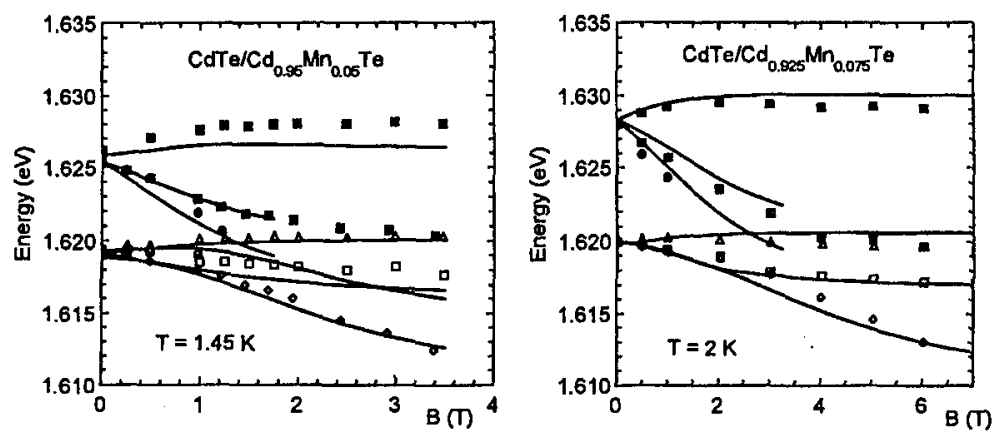

Fig. 2. Comparison of the calculated Zeeman splittings (solid lines) with those determined from measurements of the photoluminesce excitation spectra in $\mathrm{CdTe} / \mathrm{Cd}_{0.95} \mathrm{Mn}_{0.05} \mathrm{Te}$ quantum wells at $1.45 \mathrm{~K}$ [3] and in $\mathrm{CdTe} / \mathrm{Cd}_{0.925} \mathrm{Mn}_{0.075} \mathrm{Te}$ quantum wells at $2 \mathrm{~K}$ [4] for transitions from the light-hole and heavy-hole subbands to the conduction subbands.

quantum well structures characterized by variable magnetic ion concentration in layers close to the nonmagnetic quantum well.

We would like to thank P. Gassot and J. Genoe for illuminating discussions on the confinement energy levels. II.B. is also grateful to Ministère de l'Education Nationale, de l'Enseignement Superieur et de la Recherche of France for granting the post-doctoral fellowship.

\section{References}

[1] J.A. Gaj, W. Grieshaber, C. Bodin-Deshayes, J. Cibert, G. Feuillet, Y. Merle d'Aubigné, A. Wasiela, Phys. Rev. B 50, 5512 (1994).

[2] H. Bednarski, J. Cisowski, Phys. Rev. B 48, 5113 (1993).

[3] P. Peyla, A. Wasiela, Y. Merle d'Aubigné, D.E. Ashenford, B. Lunn, Phys. Rev. B 47, 3783 (1993).

[4] S.R. Jackson, J.E. Nicholls, W.E. Hagston, F. Harrison, T. Stirner, J.II.C. Hogg, B. Lunn, D.E. Ashenford, Phys. Rev. B 50, 5392 (1994).

[5] W.J.M. de Jonge, H.J.M. Swagten, J. Magn. Magn. Mater. 100, 322 (1991).

[6] J. Genoe, K. Fobelets, C. Van Hoof, G. Borghs, Phys. Rev. B 52, 14025 (1995).

[7] T. Stirner, P. Harrison, W.E. Hagston, J.P. Goodwin, Surf. Sci. 313, 417 (1994). 